\title{
Mitgliederversammlung LV Baden-Württemberg
}

Die Mitgliederversammlung des Landesverbands Baden-Württemberg fand am 25.10.15 im Marienhospital Stuttgart statt. Nach einem musikalischen Einstieg war der Vormittag aktuellen berufspolitischen Themen, wie der Einrichtung einer Pflegekammer in Baden-Württemberg und Aktivitäten zum Thema Migration und Pflege, gewidmet. Die Vorstandsmitglieder Reinhard Dummler, Barbara Driescher und Kirsten Heiland berichteten von der Vorstandsarbeit, den Aktivitäten im Landespflegerat und Kontakten zum Sozialministerium. Zur Pflegekammer wurde eine Postkartenaktion des Landespflegerats Baden-Württemberg vorgestellt: Alle Abgeordneten des Landtags erhalten in den nächsten Wochen Postkarten mit dem Motto „Wir wollen die Pflegekammer”.

Ein weiterer Schwerpunkt war der Austausch zu schulspezifischen Themen, wie Teilzeitausbildung, Auslandspraktika in der Ausbildung und Sprachförderung. Günter Schmeiser, Mitglied der arbeitsrechtlichen Kommission des Caritasverbandes, berichtete über die neue Eingruppierung für Lehrende an Pflegebildungseinrichtungen im Geltungsbereich des AVR. Am Nachmittag stellte

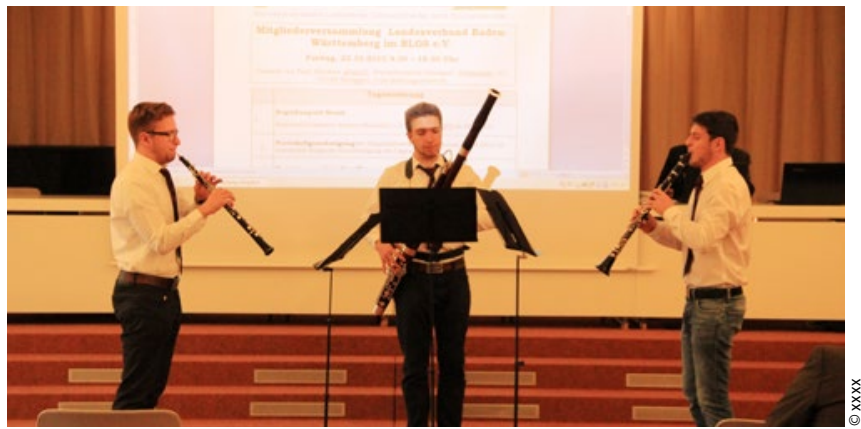

Musikalische Eröffnung der Mitgliederversammlung

Michael Breuckmann zudem den aktuellen Stand zum Pflegeberufsgesetz und die diesbezüglichen Aktivitäten des Bundesvorstands vor.

Kirsten Heiland

\section{Nordrhein-Westfalen: „Die generalistische Pflegeausbildung im Diskurs”}

Das Highlight des BLGS-NRW im Herbst war die Fachtagung am 22.10.2015 in den Westfalenhallen Dortmund mit über 150 Teilnehmern. Vor dem Hintergrund der aktuellen Diskussionen des Pflegeberufsgesetzes wurde die "Generalistische Pflegeausbildung im Diskurs" thematisiert.

Eröffnet wurde die berufspolitische Diskussion mit einem Vortrag von Prof. Dr. Frank Weidner, dip, Köln. Eindrucksvoll beschrieb er den Weg der generalistischen Pflegeausbildung und verdeutlichte die Zusammenführung der drei pflegerischen Berufe. Eine gegenteilige Position vertrat Prof. Dr. Martina Hasseler, Ostfalia Hochschule Wolfsburg. Sie bezog sich u.a. auf die Schwächen der durchgeführten Modellversuche hierzulande und verdeutlichte offen gebliebene Fragen zum Beispiel zu den "Auswirkungen auf die Qualität der Versorgung". Die unterschiedlichen Positionen waren Grundlage für eine lebhafte Diskussion im Plenum. Auf diese Weise war ein umfassender Einblick in die Vor- und Nachteile der generalistischen Pflegeausbildung möglich. Dieser unterstützte die sich anschließende Podiumsdiskus-

\section{Generalistik auch Thema beim LV Hessen}

Am 5.10.2015 veranstaltete der Landesverband Hessen im BLGS seine jährliche Fachtagung und Mitgliederversammlung in Kassel. An der Veranstaltung "Generalistik - Königs- oder Irrweg?" nahmen mehr als 50 Pflegelehrerinnen und -lehrer teil, informierten sich aus erster Hand über den Fortgang des Verfahrens zum neuen Pflegeberufsgesetz und erlebten eine spannende Diskussion rund um die generalistische Ausbildung.

Volker Pape als Gastgeber und Mitglied des Landesvorstands Hessen und Carsten Drude als Vorsitzender des Bundesvorstands eröffneten die gut besuchte Veranstaltung. Unter dem Motto „Pflegepolitik 2.0" analysierte Drude zunächst die aktuelle Legislaturperiode aus der Perspektive der professionell Pflegenden und der Pflegelehrenden. Anschließend referierte der Vorsitzende des Gesundheitsausschusses im Deutschen Bundestag Dr. Edgar Franke (MdB) sion, in der die unterschiedlichen Positionen auch in zahlreichen Wortmeldungen aus dem Plenum deutlich wurden.

Ein weiterer inhaltlicher Schwerpunkt der Tagung war die Qualitätsentwicklung an Bildungseinrichtungen. Hierzu wurde von Prof. Marcellus Bonato, Fachhochschule Münster, ein Einblick in Qualitätsmanagementsysteme und Indikatoren der Qualitätssicherung in Bildungseinrichtungen gegeben. Das Ministerium für Gesundheit, Emanzipation, Pflege und Alter NRW wurde an diesem Tag von Dr. Christine Riesner vertreten. Sie berichtete über die aktuellen politischen Entwicklungen.

Auf der anschließenden Mitgliederversammlung des BLGSNRW wurde der Vorstand mit einstimmigem Votum entlastet. Viele Anwesende lobten die Fachtagung des BLGS NRW als eine Veranstaltung, in der es gelungen sei, aktuelle Themen zu präsentieren und damit den Bedürfnissen der Mitglieder zu entsprechen.

Daniela Schlosser

über den Stand der Entwicklung des neuen Pflegeberufsgesetzes. Er thematisierte Kernanliegen und Eckpunkte der geplanten Reform und insbesondere Aspekte der zukünftigen Finanzierung.

Sandra Altmeppen berichtete aus dem abgeschlossenen Modellprojekt zur generalistischen Ausbildung an der WannseeSchule Berlin. Als Leiterin der AG Curriculum erläuterte sie anhand des Berliner Modells, wie der Weg zur Generalistik in der Ausbildungspraxis erfolgreich beschritten werden kann. Volker Pape warf abschließend einen kritischen Blick auf noch ungeklärte Aspekte und Fallstricke einer generalistischen Ausbildung und brachte einige konträre Positionen ins Spiel. Eine angeregte Diskussion rundete die gelungene Veranstaltung ab.

Angelika Unger 